\title{
Modulation of Sleep Quality and Autonomic Functioning by Symptoms of Depression in Women with Irritable Bowel Syndrome
}

\author{
JENNIFER J. T. ROBERT, PhD, ${ }^{*} \S$ WILLIAM C. ORR, PhD, $†$ and SIGRID ELSENBRUCH, PhD $\ddagger$
}

\begin{abstract}
The objective of this study was to determine how depressive symptoms affect autonomic activity during sleep, objective and subjective sleep, and gastrointestinal symptom severity in women with irritable bowel syndrome (IBS). Seventy women who met the Rome II criteria for IBS and 21 healthy volunteers participated. All participants were recruited from the surrounding community. IBS patients were stratified into two groups based on their Beck Depression Inventory II score and 44 IBS patients with depressive symptoms (IBS+DS) were compared to 26 IBS patients without depressive symptoms (IBS-DS). Autonomic activity was measured by heart rate variability (HRV) analysis. Fifteen-minute segments were selected from a baseline presleep period, stage 2, slow-wave sleep, and rapid-eye movement sleep for heart rate variability spectral analysis. Subjective sleep quality was assessed by the Pittsburg Sleep Quality Index (PSQI) and gastrointestinal symptom severity was assessed by an 18 -item questionnaire. The IBS+DS group reported significantly $(P<0.01)$ more sleep complaints, measured by the PSQI, than the IBS-DS group and healthy controls. The IBS+DS group took significantly $(P<0.05)$ longer to enter their first rapid-eye movement period than healthy controls. The IBS $+\mathrm{DS}$ group reported significantly $(P=0.01)$ increased gastrointestinal symptom severity compared to the IBS-DS group. There were no significant group differences in autonomic activity during the baseline presleep period or sleep stages. The results demonstrated that IBS patients with significant depressive symptoms had increased gastrointestinal symptom severity, increased sleep complaints, and alterations in sleep architecture compared to healthy controls and IBS patients without significant depressive symptoms.
\end{abstract}

KEY WORDS: irritable bowel syndrome; sleep; depression; anxiety; autonomic activity; heart rate variability.

In recent years, a shift in the conception of irritable bowel syndrome (IBS) has occurred from a disease-based reductionistic model to a biopsychosocial model of illness

\footnotetext{
Manuscript accepted April 28, 2004.

From the $*$ Department of Psychiatry, University of Michigan, Ann Arbor, Michigan, USA, $†$ Thomas N. Lynn Institute for Healthcare Research, Oklahoma City, Oklahoma, USA, and $\ddagger$ University Clinic of Essen, Department of Medical Psychology, Essen, Germany. §Dr. Robert is currently at the University of Michigan. Dr. Robert's former name was Dr. Jennifer J. Thompson.

Address for reprint requests: Jennifer J. T. Robert, PhD, Department of Psychiatry, University of Michigan, Sleep and Chronophysiology Laboratory, 2101 Commonwealth, Suite D, Ann Arbor, Michigan 48105, USA; jentho@med.umich.edu.
}

$(1,2)$. The biopsychosocial model integrates psychosocial factors such as stress and psychological state with the physiological mechanisms thought to be involved in IBS $(1,3)$. According to the model, psychosocial factors influence IBS symptoms, clinical outcome, and treatment (4). A large proportion of IBS patients also meets the criteria for a psychiatric disorder, mostly anxiety and/or depression, and varies according to whether the sample was from a tertiary center or a community sample.

Sleep disturbance is a common symptom of depression and IBS. Research from our group, as well as others, has demonstrated that women with IBS report more sleep disturbances than healthy controls (9-14). Elsenbruch 
et al. investigated objective polysomnographic sleep measures to determine if the sleep complaints were accompanied by changes in sleep architecture $(9,10)$. They found that the sleep complaints were not mirrored by objective measures; women with IBS had sleep architecture similar to that of healthy controls. Moreover, these results were confirmed in a subsequent investigation by the same group (14). Elsenbruch and colleagues also investigated the role of psychological disturbances on the misperception of sleep in women with IBS (10). Because the primary objective of the previous investigations was to determine the relationship of sleep complaints to objective PSG measures, individuals with moderate to severe depression, as measured by the Beck Depression Inventory (BDI-II), were excluded. Despite the exclusion of those individuals, IBS patients had increased psychological disturbances with elevated depression scores. Results indicated that the severity of psychological disturbance was significantly correlated with subjective sleep complaints (10). Thus, it appeared that psychological disturbances in IBS patients contribute to self-reported sleep complaints. The difference in sleep between IBS patients with concurrent depressive symptoms and patients without depressive symptoms has not been investigated.

Autonomic abnormalities have been detected in both IBS and depression (13-19). Orr and colleagues have conducted a series of investigations of cardiac autonomic regulation during sleep in IBS patients $(13,14)$. They determined that IBS is associated with sympathetic dominance during rapid-eye movement (REM) sleep in a group of IBS patients (20\% constipation-predominant, $80 \%$ alternating constipation/diarrhea). In a subsequent study, women with IBS who did not have dyspeptic symptoms were found to have elevated sympathetic dominance during REM sleep compared to women with IBS with dyspeptic symptoms and controls (14). The increase in sympathetic dominance in these studies was attributed to vagal withdrawal. A seminal study was conducted by Iovino et al. demonstrating that the autonomic nervous system can modulate visceral perception in healthy controls (15). They induced sympathetic activation via lower body negative pressure and found that responses to duodenal stimulation were sensitized.

Decreased heart rate variability (HRV) and increased sympathetic dominance during daytime or averaged 24-hr recordings have been found in persons with major depression compared to nondepressed controls $(16,17)$. A similar study to the present was conducted by Jarrett et al., examining HRV across 24-hr periods in women with IBS who had anxiety and depressive disorders (18). They demonstrated lower vagal tone across 24 -hr periods in women with IBS who had clinically diagnosed depres- sion compared to a group of women with IBS who did not have affective disorders. Another study reported higher heart rates during sleep in depressed patients compared to controls, but HRV was not utilized to determine autonomic activity (19). To date, there has not been an investigation of HRV during sleep stages utilizing polysomnography (PSG) in individuals with IBS and concurrent depression.

The primary hypothesis was to determine if depressive symptoms in women with IBS were associated with sympathetic dominance during REM sleep previously found in women with IBS. If the results confirm the hypothesis, then depression may be a significant factor mediating autonomic dysfunction in women with IBS. The secondary goal was to determine how significant depressive symptoms affect objective and subjective sleep and gastrointestinal symptom severity in women with IBS.

\section{MATERIALS AND METHODS}

\section{Recruitment and Screening of Participants}

The participants were recruited from the surrounding community through public advertisement. Each participant underwent a screening process that involved a structured telephone screening and a personal interview. Women with symptoms of IBS described their gastrointestinal symptoms during the telephone screening, but a more comprehensive description was obtained when they completed the Rome II integrative questionnaire at the time of the personal interview (20). Women with IBS symptoms were eligible to participate if they met the Rome II criteria for IBS and were diagnosed by a gastroenterologist within a year prior to the study. In the event that a potential participant had not been diagnosed within the past year, she was interviewed by a gastroenterologist to establish a diagnosis of IBS. Women who had hypertension, neurological disorders, cardiovascular disorders, sleep disorders, fibromyalgia or who were night-shift workers or currently pregnant were excluded from participation. Healthy controls were excluded for the presence of gastrointestinal symptoms ( $>3$ times per week) during the last 2 weeks and significant depressive symptoms (BDI $\geq 12)$ (21). Reliability and validity studies have determined that a cutoff score of 12 to 13 recognizes clinically significant depression (22). Antidepressant medication taken within the past 90 days was exclusionary. Menopausal status was not considered exclusionary and phase of menstrual cycle was not controlled. The study protocol was approved by the Institutional Review Board of the University of Oklahoma Health Sciences Center and informed consent was obtained from each participant during the personal interview.

\section{Procedures and Experimental Protocol}

The participants were stratified into two groups based on their self-reported depressive symptoms, measured by the Beck Depression Inventory II (BDI-II). Women with substantial depressive symptoms, defined as scoring 12 or higher on the BDI-II (IBS+DS), were compared to a group of women without depressive symptoms, scoring lower than 12 on the 
BDI-II (IBS-DS) and healthy controls. The BDI-II was completed during the personal interview, in conjunction with several other questionnaires: a medical history and demographic questionnaire, the Pittsburg Sleep Quality Index (PSQI), the Spielberger State Anxiety questionnaire, and an 18-item gastrointestinal symptom questionnaire to determine gastrointestinal symptom severity and frequency $(10,23,24)$. Each participant completed the 90-item Symptom Checklist Revised (SCL-90-R) the night of their polysomnographic (PSG) sleep study (25). Following the personal interview, the participants were scheduled for their polysomnography (PSG) sleep study.

All participants underwent one overnight PSG sleep study. Participants arrived at the laboratory on the day scheduled for their PSG study at approximately 8:30 PM. They refrained from medications (e.g., $\mathrm{H}_{2}$ blockers, medications causing drowsiness) for a minimum of $48 \mathrm{hr}$ prior to the PSG recording. They also abstained from caffeine for at least $6 \mathrm{hr}$ and nicotine for at least $1 \mathrm{hr}$ before reporting to the laboratory. They were instructed to consume their dinner prior to 6:00 PM and to refrain from any food and any beverages other than water after 6:00 PM.

\section{Measures and Data Acquisition}

Subjective Sleep Quality. The PSQI assessed subjective sleep quality of the past 4 weeks and was administered during the personal interview. The PSQI was scored according to the guidelines provided by the authors (23). The PSQI instrument produces a global score and seven subscale dimensions (subjective sleep quality, latency, disturbances, efficiency, duration, sleeping medication, daytime disturbance). A higher score indicates more sleep complaints.

Sleep Architecture. A standard PSG recording was completed including the EEG (central channels $\mathrm{C} 3$ and $\mathrm{C} 4$, occipital channels $\mathrm{O} 1$ and $\mathrm{O} 2$, and references $\mathrm{A} 1, \mathrm{~A} 2$, and $\mathrm{CZ}$ ), electrooculogram (EOG) (outer canther of each eye), and submental electromyogram (EMG). In order to detect any respiratory disorder, an air pressure transducer with cannula was utilized, as well as pulse oximetry and chest and abdominal effort recordings. The PSG recordings utilized digital Grass amplifiers and the EEG signals were sent to a computer system with Grass software for visual sleep stage determination. PSG recordings were blindly scored according to internationally accepted sleep staging criteria (26).

Autonomic Measures. Autonomic activity was determined by heart rate variability (HRV) spectral analysis of the beat-tobeat cardiac intervals $(27,28)$. HRV data were derived from the ECG signal utilizing standard silver/silver chloride cutaneous electrodes which were applied to the lower left side of the thorax on the midaxillary line and on the right and left shoulder over the clavicle to detect the ECG signal. A single channel of ECG was recorded at a sampling rate of $6000 \mathrm{~Hz}$ and 16-bit resolution and analyzed after being downsampled to $500 \mathrm{~Hz}$. The internal clock of the computer recording the ECG signal was synchronized with the computer recording the PSG in order to allow epoch-by-epoch matching of ECG with sleep stages and events. The R-R intervals were calculated, resampled, and interpolated by computer software, yielding the HRV signal for 15-min periods of a presleep waking period and stage 2 , slow-wave, and REM sleep. The 15-min segments were analyzed using the digital signal processing toolbox of MATLAB to compute fast Fourier transforms (Math Works Inc., Natick, MA) (29).

Autonomic functioning was assessed by the power of specific frequency bands in the spectral analysis of HRV (30). The percent high-frequency (HF) power band was a marker of vagal tone defined as the percent power in the $0.15-$ to $0.50-\mathrm{Hz}$ frequency range. The percent low-frequency (LF) band power was defined as $0.04-0.15 \mathrm{~Hz}$ and can include both vagal and sympathetic influences, thus sympathetic activation is often inferred from the $\mathrm{LF} / \mathrm{HF}$ ratio (31). The $\mathrm{LF} / \mathrm{HF}$ ratio was considered an indicator of sympathovagal balance $(14,28)$. Each segment was analyzed by spectral analysis to calculate the percentage LF band power, percent $\mathrm{HF}$ band power, and LF/HF ratio.

\section{Statistical Analyses}

Multivariate analysis of variance (MANOVA) was conducted to detect group differences for quantitative data. Twotailed independent $t$-tests determined mean differences for IBS groups (IBS+DS, IBS-DS). Frequency data were analyzed by Pearson's chi-square analysis. Alpha level was set at 0.05 for all statistical analyses. The Bonferroni correction was utilized to adjust alpha for multiple comparisons.

\section{RESULTS}

All results are shown as mean \pm standard error of the mean (SE). Age and body mass index were used as covariates in the HRV analyses.

\section{Demographic Characteristics}

The IBS+DS group had a significantly higher body mass index (BMI) and were older (Table 1) than the IBS-DS group and healthy controls. The BDI-II means for the IBS-DS and IBS+DS groups were statistically significantly different, 4.9 and 20.2, respectively. The frequency distribution is illustrated in Figure 1. Sixty-three (69\%) women were premenopausal and 28 (31\%) women were postmenopausal, but the difference was not statistically significant. A total of 15 women were taking hormone replacement therapy and the distribution was not significantly different by group, nor was phase of menstrual cycle different by group for the premenopausal women.

\section{Subjective Sleep Quality}

The overall MANOVA for subjective sleep quality detected significant group differences on the PSQI $(F[14,160]=5.79, p<0.01)$. Individual ANOVAs for each PSQI dimension also revealed significant $(P<0.01)$ group differences on each subscale, with IBS+DS revealing more dysfunction in every dimension (see Figure 2). Multiple comparisons revealed that the IBS + DS group had significantly $(P<0.05)$ higher global PSQI scores $(11.5 \pm 0.5)$ than the IBS-DS group $(8.0 \pm 0.7)$ and healthy controls $(3.1 \pm 0.3)$. The IBS-DS group also 
TABle 1. PARTICIPANT CHARACTERISTICS

\begin{tabular}{|c|c|c|c|c|}
\hline & $\begin{array}{l}I B S-D S \\
(\mathrm{n}=44)\end{array}$ & $\begin{array}{l}I B S+D S \\
(\mathrm{n}=26)\end{array}$ & $\begin{array}{l}\text { Healthy controls } \\
\quad(\mathrm{n}=21)\end{array}$ & $\mathrm{P}$ value \\
\hline Age, years* & $33.0 \pm 1.1$ & $40.1 \pm 2.6 \dagger$ & $34.7 \pm 1.9$ & 0.01 \\
\hline \multicolumn{5}{|l|}{ Race $(n)$} \\
\hline African American & 3 & 2 & 0 & \\
\hline Caucasian & 39 & 20 & 19 & \\
\hline Native American & 1 & 2 & 1 & NS \\
\hline Hispanic & 0 & 1 & 1 & \\
\hline Asian & 1 & 1 & 0 & \\
\hline Body mass index, $\mathrm{kg} / \mathrm{m}^{2} *$ & $25.0 \pm 0.9$ & $28.9 \pm 1.2 \dagger$ & $24.2 \pm 0.7$ & 0.01 \\
\hline Postmenopausal $(n)$ & 9 & 12 & 7 & NS \\
\hline Postmenopausal, taking hormone replacement therapy $(n)$ & 5 & 6 & 4 & NS \\
\hline Premenopausal, taking oral contraceptives $(n)$ & 14 & 3 & 5 & NS \\
\hline Follicular phase $(n)$ & 9 & 15 & 7 & \\
\hline Ovulation phase $(n)$ & 15 & 2 & 5 & NS \\
\hline Luteal phase $(n)$ & 9 & 3 & 2 & \\
\hline
\end{tabular}

Note. Some of the columns do not sum to the total number of cases because of missing data. NS, nonsignificant. *Data are mean $\pm \mathrm{SE}$.

$\dagger$ IBS+DS significantly $(P<0.05)$ greater than IBS - DS and controls.

had significantly $(P=0.01)$ higher PSQI scores than the healthy controls.

\section{Sleep Macroarchitecture}

No respiratory disturbances were found in any of the participants. The IBS+DS group took significantly $(P=0.04)$ longer to enter their first REM period than the healthy controls (see Table 2). There was a trend $(P=0.08)$ for prolonged REM latency in the IBS-DS group compared to healthy controls. No other group differences in sleep macroarchitecture were found.

\section{Psychological Measures}

A one-way ANOVA indicated that the IBS+DS group had significantly $(F[2,74]=8.21, \quad P<0.01)$ higher state anxiety than both the IBS-DS group $(P<$ $0.01)$ and the healthy controls $(P<0.01)$. The increased anxiety in the IBS+DS group was not significantly correlated $(r=0.03, P=0.88)$ with their prolonged REM sleep latency. A MANOVA determined that the IBS+DS group scored significantly $(P<$ $0.05)$ higher than the IBS-DS group and healthy controls on the SCL-90-R global scores for psychological

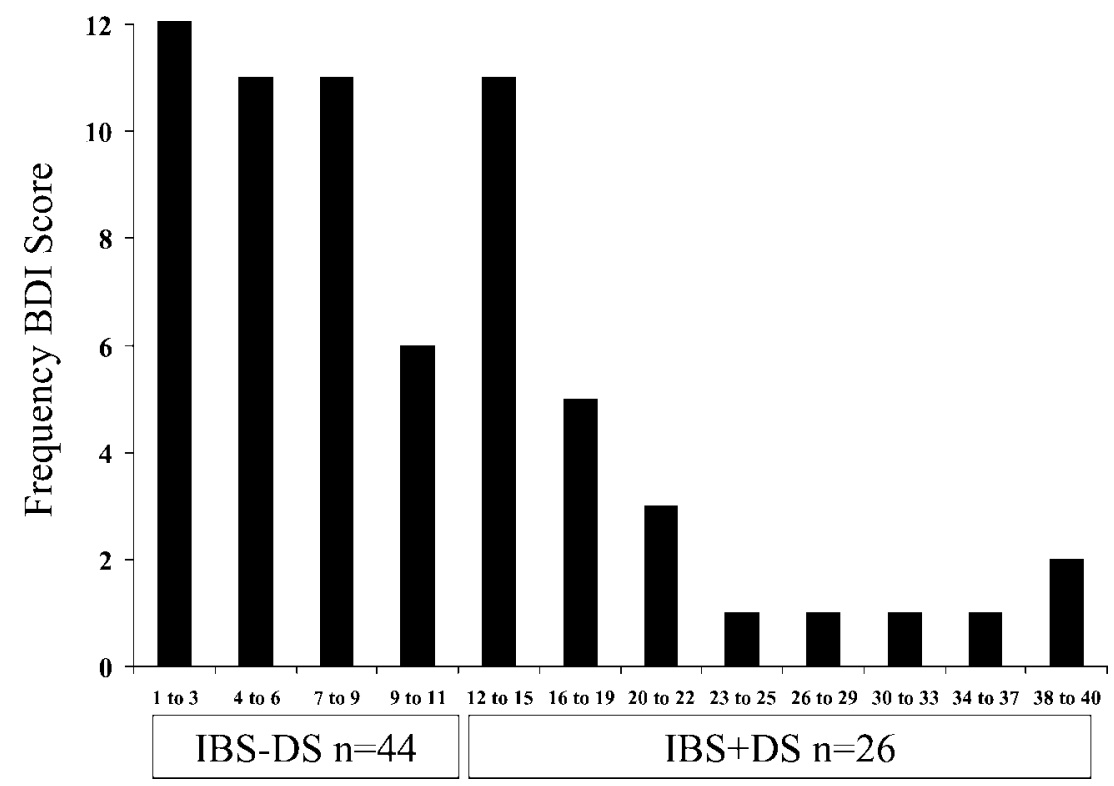

Fig 1. Beck Depression Inventory II Frequency Distribution. The IBS - DS group had significantly lower BDI-II scores (4.9) than the IBS+ group (20.2). 


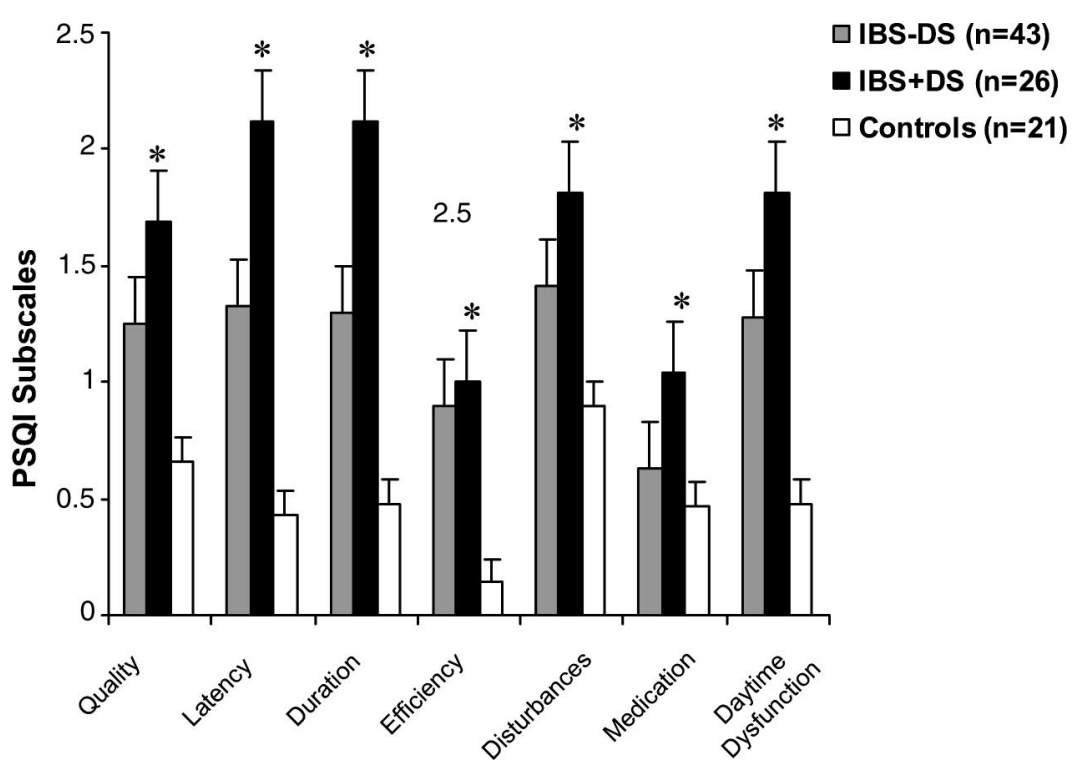

Fig 2. Subjective sleep quality, PSQI global score. The IBS+DS group reported significantly $(* P<0.01)$ more sleep complaints on each of the PSQI subscales (global scores not shown) than the IBS-DS group or controls. Bonferroni alpha adjustments were made. The data are mean and SE.

disturbance as well as all of the subscales (see Figure 3).

\section{Autonomic Activity-Heart Rate Variability}

Two individuals were eliminated from the analysis due to artifacts (e.g., movement) occurring in the 15-min REM segment. A MANOVA was conducted to determine group differences during each 15 -min segment (baseline presleep period and stage 2, slow-wave, and REM sleep) for the LF/HF ratio during REM sleep. The overall $F$-test $(F[4,77]=1.05, P=0.38)$ did not indicate any significant group differences (see Figure 4). Two separate MANOVAs were conducted to detect any group differences in percent LF band power and percent
HF band power and there were no significant group differences $(F[4,66]=1.7, P=0.15$, and $F[4,66]=1.8$, $P=0.16$, respectively).

\section{Gastrointestinal Symptom Profile}

Nineteen of the IBS patients were constipationpredominant, 31 IBS patients were diarrhea-predominant, and 20 patients alternated between constipation and diarrhea symptoms. A Pearson's chi-square did not detect unequal distributions between the groups (Table 3). All participants had similar reports of lower abdominal pain approximately 4 days a week. The groups reported having had their IBS symptoms for similar durations. As

TABle 2. Polysomnographic Parameters

\begin{tabular}{lcccc}
\hline & $\begin{array}{c}I B S-D S \\
(\mathrm{n}=44)\end{array}$ & $\begin{array}{c}I B S+D S \\
(\mathrm{n}=26)\end{array}$ & $\begin{array}{c}\text { Healthy controls } \\
(\mathrm{n}=21)\end{array}$ & P value \\
\hline Total sleep time (TST) (min) & $378.9 \pm 7.7$ & $374.0 \pm 11.0$ & $378.7 \pm 8.9$ & NS \\
Time in bed (TIB) (min) & $442.5 \pm 5.2$ & $446.3 \pm 7.3$ & $439.8 \pm 5.7$ & NS \\
Sleep onset latency (min) & $23.9 \pm 2.9$ & $30.2 \pm 6.3$ & $22.7 \pm 3.5$ & NS \\
Sleep efficiency (TST/TIB) & $85.6 \pm 1.5$ & $83.7 \pm 2.0$ & $86.1 \pm 1.6$ & NS \\
Number of awakenings after sleep onset & $7.7 \pm 0.7$ & $8.6 \pm 1.1$ & $9.1 \pm 1.2$ & NS \\
Stage 2 (\%) & $56.6 \pm 1.3$ & $58.2 \pm 1.7$ & $56.8 \pm 1.6$ & NS \\
Stages 3 \& 4 (slow-wave sleep; \%) & $17.2 \pm 1.1$ & $17.3 \pm 1.6$ & $16.3 \pm 1.4$ & NS \\
REM sleep (\%) & $21.7 \pm 0.9$ & $21.6 \pm 0.9$ & $22.3 \pm 0.9$ & NS \\
REM onset latency (min) & $122.5 \pm 10.5$ & $126.3 \pm 12.9 *$ & $84.9 \pm 9.4$ & 0.04 \\
REM episodes & $3.7 \pm 0.2$ & $3.8 \pm 0.3$ & $3.8 \pm 0.2$ & NS
\end{tabular}

Note. Data are mean \pm SE. NS, nonsignificant; REM, rapid eye movement.

*IBS+DS significantly $(P<0.05)$ greater than controls after Bonferroni alpha adjustment. 


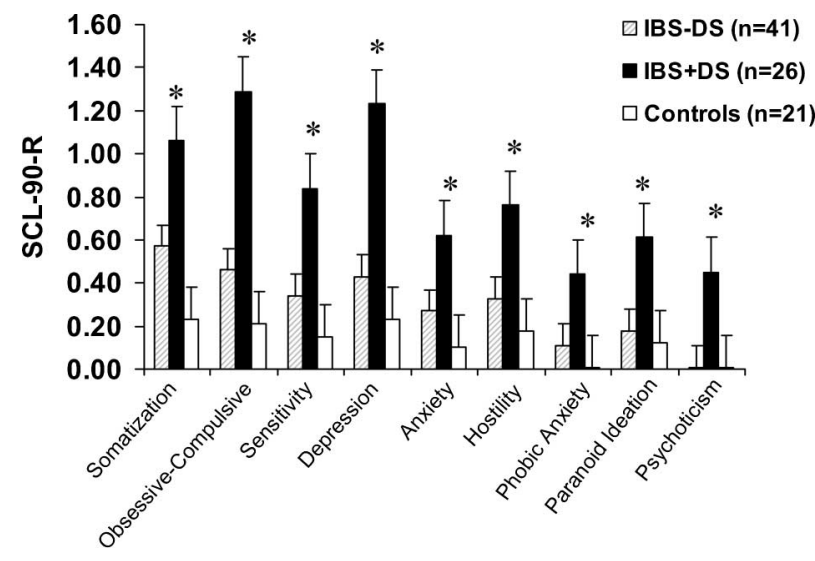

Fig 3. Psychological disturbance subscales (SCL-90-R). The IBS+DS group had significantly $(* P<0.01)$ higher scores on each dimension of the SCL-90-R than the IBS-DS group and controls. Bonferroni alpha adjustments were made. The data are shown as mean and SE.

illustrated in Table 3, a majority (not statistically significant) of participants (96 vs. 68\%) in the IBS+DS group reported that their abdominal pain prevented them from sleeping. Analysis of the 18-item gastrointestinal questionnaire revealed that the IBS+DS group reported significantly $(t=2.08$, df $=68, P=0.01$ ) more gastrointestinal symptom severity than the IBS-DS group.

\section{DISCUSSION}

The present data extend our previous work on the nature of sleep complaints in women with IBS, which demonstrated that women with IBS rated their sleep quality significantly worse than healthy controls $(9,10)$. Previously, we could not determine how much depressive symptoms

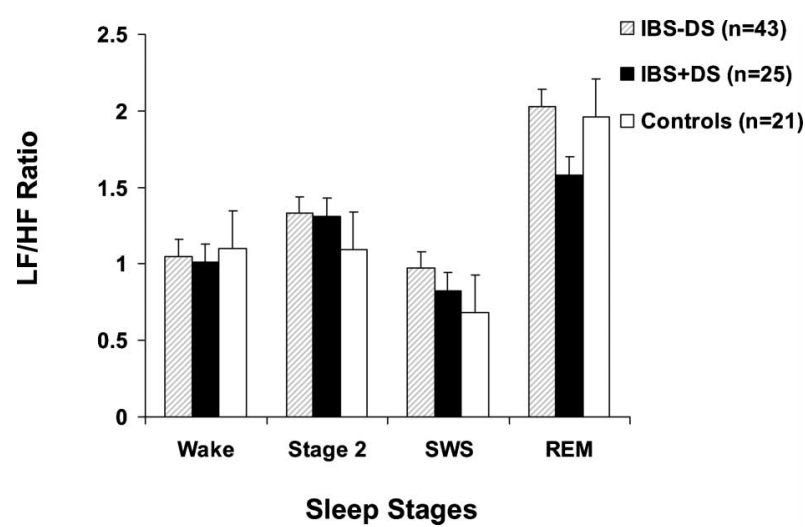

Fig 4. Autonomic activity across sleep stages. There were no significant group differences in sympathetic dominance during sleep stages. The raw data, without age and BMI adjustments, are shown as mean and SE. affected subjective sleep complaints in IBS since depressive symptoms were exclusionary. Sleep complaints are common in depression, thus we hypothesized that depressive symptoms in IBS would be associated with greater sleep complaints. Indeed, the present data convincingly confirm that sleep complaints in IBS are dramatically affected by the presence of depressive symptoms. The women with IBS and depressive symptoms had significantly greater sleep complaints than the women with IBS without depressive symptoms as well as the healthy controls.

Women with IBS and depressive symptoms reported significantly more state anxiety than women with IBS without depressive symptoms. To decipher the relationship between anxiety and depression on subjective sleep measures, we conducted an ANOVA utilizing anxiety as a covariate. Results showed that subjective sleep complaints remained significantly greater in the IBS group with depressive symptoms compared to the IBS group without depressive symptoms. This suggests that depressive symptoms are important determinants of increased sleep complaints in women with IBS and are not modulated by the presence or absence of anxiety symptoms.

Subjective sleep complaints in women with IBS were not substantiated by objective alterations in sleep architecture (e.g., efficiency, number of awakenings, or percentage of slow-wave sleep) in two of our earlier investigations $(9,10,13,14)$. The significantly longer REM latency in IBS patients with depressive symptoms, compared to IBS patients without depressive symptoms and controls, had not been found in any of our previous investigations. However, our previous studies excluded individuals with depression. There have been two other studies that did not exclude depressive symptoms in IBS and found prolonged REM latency compared to controls $(12,32)$. This suggests that increased REM latency may be attributable to the concurrent presence of depressive symptoms in IBS. The presence of anxiety was not significantly correlated with REM latency for any group.

The results from the objective sleep data may have been affected by a first-night effect since participants slept in the laboratory for 1 instead of 2 nights. The reasons for this relate not only to issues of cost and feasibility but also to the fact that previous studies have shown only minor differences between consecutive sleep studies (33). An important factor in minimizing the first-night effect is to facilitate the participants comfort in the sleep laboratory (34). To maximize their comfort, each participant had her own private bedroom with all of the necessary furnishings (extra pillows and blankets, fan, mirror, dresser vanity, full-size mattress). In addition, there was a call button to 
TABle 3. IBS Symptom PROFILE

\begin{tabular}{lccc}
\hline & $\begin{array}{c}I B S-D S \\
(\mathrm{n}=44)\end{array}$ & $\begin{array}{c}I B S+D S \\
(\mathrm{n}=26)\end{array}$ & P value \\
\hline Predominant symptom & & & \\
$\quad$ Constipation & 23 & 8 & $\mathrm{NS}$ \\
$\quad$ Diarrhea & 9 & 10 & \\
$\quad$ Alternating & 12 & 8 & \\
Days with pain/week & $4.2 \pm 0.3$ & $3.8 \pm 0.3$ & $\mathrm{NS}$ \\
>5 years suffered with pain, \% & 51 & 57 & $\mathrm{NS}$ \\
Abdominal bloating, \% & 90 & 96 & $\mathrm{NS}$ \\
Abdominal bloating/week & $3.9 \pm 0.3$ & $3.6 \pm 1$ & $\mathrm{NS}$ \\
Abdominal pain prevents falling asleep, \% yes & 68 & 96 & $\mathrm{NS}$ \\
Abdominal pain awakens from sleep, \% yes & 78 & 96 & $\mathrm{NS}$ \\
Gastrointestinal symptom severity & $1.4 \pm 0.1$ & $1.7 \pm 0.1^{*}$ & 0.04 \\
Excessive passing of gas by rectum & $0.93 \pm 0.2$ & $1.6 \pm 0.2^{*}$ & 0.01 \\
Overly full or bloated & $1.3 \pm 0.2$ & $2.1 \pm 0.2^{*}$ & 0.01 \\
Incomplete bowel movement $>$ 25\% of the time & $1.4 \pm 0.2$ & $2.2 \pm 0.2^{*}$ & 0.01 \\
Nausea & $0.9 \pm 0.1$ & $1.4 \pm 0.2^{*}$ & 0.03 \\
Strain $>$ 25\% of the time during a bowel movement & $1.6 \pm 0.1$ & $2.1 \pm 0.2^{*}$ & 0.05 \\
\hline
\end{tabular}

Note. Some of the columns do not sum to the total number of cases of missing data. NS, nonsignificant. *Range: 0 (never occurs) to 3 (occurs more than 2 times per week).

alert the sleep technician for any additional requests (i.e., restroom break, adjust temperature in the room). We feel it is unlikely that a "first-night effect" would appreciably alter our results.

Our previous work on sympathovagal balance across sleep stages demonstrated that compared to non-REM sleep, REM sleep is associated with an increase in sympathetic dominance, measured by the LF/HF ratio, in IBS patients compared to healthy controls $(13,14)$. The results of this study do not support any relationship between depressive symptoms and autonomic activity in women with IBS. A study similar to ours was conducted by Jarrett et al., investigating the impact of depression in IBS on autonomic functioning across 24-hr recordings (18). They reported lower vagal tone averaged across $24 \mathrm{hr}$ in women with IBS who had clinically diagnosed depression compared to women with IBS who were not diagnosed with depression. However, our inability to detect group differences for sympathovagal balance (LF/HF) is consistent with the findings of Jarrett et al. despite the methodological differences between the two studies. First, this investigation focused on the presence of depressive symptoms in IBS rather than a clinical diagnosis of depression. Second, we specifically assessed autonomic activity across sleep stages rather than averaging across a 24 -hr time period or averaging across the total sleep period $(18,35)$. To reiterate, this was the first study to investigate autonomic changes during specific sleep stages in women with IBS who have depressive symptoms. Although these findings are not consistent with the existing literature of increased sympathetic dominance in depressed patients, daytime or averaged 24-hr recordings were utilized rather than evaluating specific sleep stages verified by polysomnographic recordings $(16,17)$.

A further explanation of our findings relates to the fact that the majority of studies investigating depression and autonomic activity either have been conducted in males or have failed to control for gender differences $(16-18,36)$. There are data to show that males and females differ in HRV parameters (37). For example, Bonnemeier et al. detected shorter R-R intervals (msec) across 24-hr recordings in females compared to males and Elsenbruch et al. found increased sympathetic dominance during REM sleep in males compared to females $(38,39)$ Hence, previous reports of increased sympathetic dominance in depression using HRV may be confounded by gender differences. Clearly, the interpretation of our present findings is hampered due to the lack of data on cardiac autonomic activity in males and females with clinically diagnosed major depressive disorder.

In summary, the present study represents the largest sample of IBS patients with full PSG reported to date $(n=70)$. The outcome of this study indicates that depressive symptoms in IBS affect sleep macroarchitecture and subjective sleep, but the depressive symptoms are not specifically associated with autonomic changes during sleep. Thus, these data provide substantial documentation of the importance of comorbid depression in the manifestation of both daytime and nighttime gastrointestinal symptoms as well as subjective and objective sleep in patients with IBS. 


\section{ACKNOWLEDGMENTS}

The work was supported by Grant HR02-103 from the Oklahoma Center for the Advancement of Science and Technology and the Lynn Institute for Healthcare Research, Oklahoma City. The authors would like to thank Dr. Deborah Blalock for help in confirming the patients' diagnosis and the sleep technicians of the Lynn Institute for Healthcare Research for their expertise in ensuring quality sleep recordings. The authors also wish to thank Dr. Roseanne Armitage and Dr. Robert Hoffmann for a careful and helpful review of the manuscript.

\section{REFERENCES}

1. Drossman DA, Whitehead WE, Camilleri M. Gastroenterology: Irritable bowel syndrome: A technical review for practice guideline development. Gastroenterology 112:2120-2137, 1997

2. Drossman DA: Presidential address: Gastrointestinal illness and bipsychosocial model. Psychosom Med 60;258-267, 1998

3. Gaynes BN, Drossman DA: The role of psychosocial factors in irritable bowel syndrome. Bailliere's Clin Gastroenterol 13:437-452, 1999

4. American Gastroenterological Association: AGA medical position statement: Irritable bowel syndrome. Gastroenterology 123:21052107,2002

5. Ford MJ, Miller PM, Eastwood J, et al.: Life events, psychiatric illness and the irritable bowel syndrome. Gut 28:160-165, 1987

6. Lydiard RB: Irritable bowel syndrome, anxiety, and depression: What are the links? J Clin Psychiatry 62:38-47, 2001

7. Schwartz SP, Blanchard EB, Berreman CF, et al.: Psychological aspects of irritable bowel syndrome: Comparisons with inflammatory bowel disease and nonpatient controls. Behav Res Ther 31:297-304, 1993

8. Garakani A, Win T, Virk S, et al.: Comorbidity of irritable bowel syndrome in psychiatric patients: A review. Am J Ther 10:61-67, 2003

9. Elsenbruch S, Harnish MJ, Orr WC: Subjective and objective sleep quality in irritable bowel syndrome. Am J Gastro 94:2447-2452, 1999

10. Elsenbruch S, Thompson JJ, Harnish MJ, et al.: Behavioral and physiological sleep characteristics in women with irritable bowel syndrome. Am J Gastro 97:2306-2314, 2002

11. Fass R, Fullerton S, Tung S, et al.: Sleep disturbances in clinic patients with functional bowel disorders. Am J Gastro 95:11952000,2000

12. Heitkemper M, Charman AB, Shaver J, et al.: Self-report and polysomnographic measures of sleep in women with irritable bowel syndrome. Nurs Res 47:270-277, 1998

13. Orr WC, Elsenbruch S, Harnish MJ: Autonomic regulation of cardiac function during sleep in patients with irritable bowel syndrome. Am J Gastro 98:2865-2871, 2000

14. Thompson JJ, Elsenbruch S, Harnish MJ, et al.: Autonomic functioning during REM sleep differentiates IBS symptom subgroups. Am J Gastro 97:3147-3153, 2002

15. Iovino P, Azpiroz F, Domingo E, et al.: The sympathetic nervous system modulates perception and reflex responses to gut distension in humans. Gastroenterology 108:680-686, 1995

16. Agelink MW, Boz C, Ullrich $\mathrm{H}$, et al.: Relationship between major depression and heart rate variability. Clinical consequences and im- plication for antidepressive treatment. Psychiatry Res 113:139-149, 2002

17. Carney RM, Blumenthal JA, Stein PK, et al.: Depression, heart rate variability and acute myocardial infarction. Circulation 104:20242028, 2001

18. Jarrett ME, Burr RL, Cain KC, Hertig V, Weisman P, Heitkemper MM: Anxiety and depression are related to autonomic nervous system function in women with irritable bowel syndrome. Dig Dis Sci 48:386-394, 2003

19. Volkers AC, Tulen JHM, van den Broek WW, et al.: Motor activity and autonomic cardiac functioning in major depressive disorder. $\mathrm{J}$ Affect Dis 76:23-30, 2003

20. Boyce PM, Koloski NA, Talley NJ: Irritable bowel syndrome according to varying diagnostic criteria: Are the new Rome II criteria unnecessarily restrictive for research and practice? Am J Gastro 11:3176-3183, 1995

21. Beck AT, Steer RA, Brown GK: Manual for the Beck Depression Inventory, 2nd ed. San Antonio, TX, Psychological Corp., 1996

22. Lasa L, Ayuso-Mateos JL, Vazquez-Barquero JL, Diez-Manrique FJ, Dowrick CF: The use of the Beck Depression Inventory to screen for depression in the general population: A preliminary analysis. $\mathrm{J}$ Affect Dis 57:261-265, 2000

23. Buysse DJ, Reynolds CF, Monk TH, et al.: The Pittsburgh sleep quality index: A new instrument for psychiatric practice and research. Psychiatry Res 28:193-213, 1989

24. Spielberger CD, Gorsuch RL, Lushene RE: Manual for the StateTrait Anxiety Inventory. Palo Alto, CA, Consulting Psychologists, 1970

25. Derogatis L: The SCL-90-R. Administration, Scoring and Procedures Manual II. Towson, MD, Clinical Psychometric Research, 1975,1983

26. Rechtschaffen, Kales: A Manual of Standardized Terminology, Techniques, and Scoring System for Sleep Stages of Human Subjects. Los Angeles, CA, UCLA Brain Information Service/Brain Research Institute, 1968

27. Berntson GG, Bigger JT, Eckberg DL, et al.: Heart rate variability: Origins, methods, and interpretive caveats. Psychophysiology 34:623-648, 1997

28. Kamath MV, Fallen EL: Power spectral analysis of heart rate variability: A noninvasive signature of cardiac autonomic function. Crit Rev Biomed Eng 21:245-311, 1993

29. Toolbox for MATLAB. Natick, MA, Math Works Inc.

30. Akselrod S, Gordon D, Ubel FA, et al.: Power spectrum analysis of heart rate fluctuation: A quantitative probe of beat-to-beat cardiovascular control. Science 213:220-222, 1981

31. Berger RD, Saul JP, Cohen RJ: Transfer function analysis of autonomic regulation. I. Canine atrial rate response. Am J Physiol 256:H142-H152, 1989

32. Rotem AY, Sperber AD, Krugliak P, Freidman B, Tal A, Tarasiuk A: Polysomnographic and actigraphic evidence of sleep fragmentation in patients with irritable bowel syndrome. Sleep 26:747-752, 2003

33. Kupfer DJ, Weiss, BL, Detre TP, et al.: First night effect revisited: A clinical note. J Nerv Ment Dis 159:205-209, 1974

34. Browman CP, Cartwright RD: The first night effect on sleep and dreams. Biol Psychiatry 15:809-812, 1980

35. Viola AU, Simon C, Ehrhart J, et al.: Sleep processes exert a predominant influence on the 24-h profile of heart rate variability. J Biol Rhythms 17:539-547, 2002

36. Cajochen C, Pischke J, Aeschbach D, Borbely AA: Heart rate dynamics during human sleep. Physiol Behav 55:769-774, 1994 
37. Thayer JF, Smith M, Rossy LA, Sollers JJ, Friedman BH: Heart period variability and depressive symptoms: Gender differences. Biol Psychiatry 44:304-306, 1998

38. Bonnemeier $\mathrm{H}$, Wiegand $\mathrm{U}$, Brandes A, et al.: Circadian profile of cardiac autonomic nervous modulation in healthy subjects: Differing effects of aging and gender on heart rate variability. J Cardiovasc Electrophysiol 14:791-799, 2003

39. Elsenbruch S, Harnish MJ, Orr WC: Heart rate variability during waking and sleep in healthy males and females. Sleep 22:10671071, 1999 